\section{EL SIGNIFICADO HISTÓRICO DE LAS APORTACIONES DE MARAÑÓN A LA ENDOCRINOLOGÍA INTERNACIONAL}

\author{
Carla P. Aguirre Marco \\ Instituto de Historia de la Medicina y de la Ciencia López Piñero \\ (CSIC-Universidad de Valencia) \\ aguirrep@uv.es
}

\begin{abstract}
Cómo citar este artículo/ Citation: Aguirre Marco, C.P. (2013). El significado histórico de las aportaciones de Marañón a la endocrinología internacional. Arbor, 189(759):a003. doi: http://dx.doi.org/10.3989/arbor.2013.759n1002
\end{abstract}

Recibido: 3 septiembre 2012; Aceptado: 1 octubre 2012.

RESUMEN: Una aproximación a la temprana difusión internacional de la obra endocrinológica de Marañón permite situar su protagonismo en el nacimiento de la especialidad médica en España en el marco de la endocrinología internacional, que cristalizaba en la medicina clínica europea de la misma generación de Marañón, la de 1914. Lo hacía sirviendo a una corriente renovadora que centraba de nuevo la mentalidad médica en la persona del paciente, por lo que no se concibió inicialmente la posibilidad de su escisión como especialidad médica.

PALABRAS CLAVE: Marañón; endocrinología; patología constitucional; especialización médica; Generación de 1914; Edad de Plata; España; comunicación científica internacional.

Los contenidos de la obra endocrinológica de Gregorio Marañón han venido siendo descritos y puestos en valor en sucesivas aproximaciones a lo largo de medio siglo ${ }^{1}$. La reciente puesta al día de su obra médica (ROOM, 2003) ha revelado tanto la precocidad como la vigencia de no pocas de sus aportaciones originales a la endocrinología internacional, y no sólo las más puramente clínicas sino también las científicas que han contribuido a conformar los saberes de la endocrinología actual. Por otra parte, concurrieron en

\section{THE HISTORICAL SIGNIFICANCE OF MARAÑÓN'S CONTRIBUTIONS TO INTERNATIONAL ENDOCRINOLOGY}

Copyright: (C) 2013 CSIC. Este es un artículo de acceso abierto distribuido bajo los términos de la licencia Creative Commons Attribution-Non Commercial (by-nc) Spain 3.0.

ABSTRACT: This approach to the early international dissemination of Marañón's endocrinological work highlights the leading role he played in the emergence of this medical specialty in Spain in the international context. Endocrinology was taking shape in European clinical medicine during Marañon's generation, that of 1914 , contributing to refocusing medical mind-set on the patient, such that endocrinology was initially not conceived of as an autonomous medical specialty.

KEYWORDS: Marañón; endocrinology; constitutional pathology; specialisation of medicine; Generation of 1914; Silver Age; Spain; international scientific communication.

su persona y su labor casi todas las actividades y posiciones que caracterizan el proceso inicial de especialización médica, lo que hace de Marañón el protagonista indiscutible del nacimiento de la endocrinología española.

Ambos aspectos contribuyen a mantener una imagen del endocrinólogo Marañón aislado sin atender a la tradición en la que se insertó ni a sus relaciones y colaboraciones profesionales tanto en el ámbito 
nacional como el internacional, a pesar de los estudios que proporcionan las coordenadas históricocientíficas adecuadas de su labor ${ }^{2}$. Su contribución a la endocrinología se sitúa en el marco de la medicina española de su generación, la de 1914, y su integración en el contexto internacional antes de la guerra civil española: la medicina europea del periodo de entreguerras en la que comenzaba también a cristalizar la endocrinología fuertemente impulsada desde el mundo de habla alemana de la mano de la misma generación de clínicos, la de la "preguerra" europea.

La endocrinología como especialidad "nueva" siguió el patrón institucional de las especialidades "clásicas" - la oftalmología, la otorrinolaringología, etc. bien asentado ya mediado el siglo XIX (Rosen, 1944) cuando las enfermedades endocrinas y carenciales no podían ni vislumbrarse. Junto con algunos otros nuevos focos de interés en el primer tercio del siglo XX, como la psiquiatría, la endocrinología contribuyó a renovar el pensamiento médico centrando su atención de nuevo en la persona enferma, en el paciente. Además, en la sociedad europea se venía agudizando la conciencia por los problemas sociales, incluyendo la salud, lo mismo que la convicción de que su responsabilidad recaía en el Estado lo que alcanzó gran prominencia durante la década de los años treinta (Rosen, 1974,125). En España, y en particular de la medicina de la generación de Marañón se puede afirmar lo mismo, aunque la guerra civil supuso una temprana y radical quiebra del proceso ${ }^{3}$.

En el caso de la endocrinología no pesaron otros factores sociales especiales como fueran los ideológicos y demográficos en el caso de la pediatría, o la marginación social de los enfermos en el de la dermatología, que contaba con instituciones asistenciales mucho antes de convertirse en una especialidad, lo mismo que la psiquiatría, por mencionar ejemplos conocidos de todos. Sin embargo, sí cabe destacar que, como en el caso español la guerra civil, la segunda guerra europea interrumpió el proceso de especialización dilatándolo más de dos décadas, lo suficiente para que la orientación de la propia disciplina hubiera cambiado drásticamente, como veremos.

A diferencia de las especialidades "clásicas", cuyos contenidos salieron de los tratados de medicina a las monografías especializadas, en el caso de la endocrinología las primeras monografías se adelantaron a los capítulos que habrían de incluir enseguida los manuales y tratados de medicina ${ }^{4}$. Porque, en efecto, la aparición de esas monografías es uno de los primeros signos de la especialización médica. Al que suele seguir la asociación y comunicación entre quienes se dedican a su cultivo en reuniones científicas y en nuevas sociedades, la fundación de sus revistas portavoces, hasta la aparición de puestos profesionales e instituciones especializadas, entre ellas las docentes
- como las cátedras universitarias - y las científicas y asistenciales - sean servicios hospitalarios, hospitales monográficos, institutos de investigación, etc.

Comencemos por revisar desde esta perspectiva el papel que jugara Marañón en la cristalización de la especialidad en España. Publicó uno de los primeros manuales españoles, si no el primero, de endocrinología, Las glándulas de secreción interna y las enfermedades de la nutrición (1914), que alcanzó una gran difusión, de lo que dan cuenta sus tempranas reediciones, la tercera de 1920. Siguieron dos conocidas monografías en 1915 y en 1922, ésta última una dura respuesta a los fisiólogos "puros" que cuestionaban el valor científico de las aportaciones de los clínicos y en especial su utilización de la opoterapia ${ }^{5}$. Su capítulo de endocrinología en el primer tratado español de medicina interna data de 1920 (Marañón,1920, 3-108). El segundo manual de endocrinología lo publicó en Buenos Aires en 1939 con un título que atestigua el cambio que se estaba produciendo hacia la especialización, dedicado a "las enfermedades endocrinas".

Fundó Marañón la primera revista española especializada en 1924, Archivos de endocrinología y nutrición (Madrid, 1924-28), ocupó la primera cátedra española, creada para él en 1931, fundó y presidió la Sociedad Española de Endocrinología en 1950 -integrando la ya existente Sociedad catalana de endocrinología y nutrición, algo que suele olvidarse-, lo mismo que su primer congreso celebrado en Granada en 1954, y dirigió su primera revista portavoz, Acta endocrinologica ibérica (Madrid, 1951-52) continuación de la también impulsada por Marañón Acta endocrinologica et gynaecologica hispano-lusitana (1948-1950)que pasaría enseguida a llamarse Revista ibérica de endocrinología (1952-1974) y a editarse en Barcelona, como sus sucesoras hasta hoy.

Desde sus comienzos trató Marañón de aglutinar a los diferentes grupos españoles que trabajaban en este campo, pero también a los portugueses y latinoamericanos, abriendo los espacios comunes mencionados y promoviendo las visitas e intercambios de investigadores. Su primera revista la codirigió con Roberto Nóvoa Santos (1885-1933), al frente de su grupo entonces en Santiago de Compostela, Augusto Pi Sunyer (1879-1965) representando al grupo catalán, y Bernardo A. Houssay (1887-1971) de Buenos Aires, con Enrique Carrasco Cadenas ${ }^{6}$ al frente de la redacción. Las siguientes revistas fueron órganos de expresión tanto de la sociedad española como de la portuguesa de endocrinología e integraban en su redacción, junto con los portugueses, a endocrinólogos de Madrid y Barcelona primero, a los que se sumaron enseguida de Valladolid y de Granada.

Esta labor de Marañón discurría a la par que en el resto de Europa. En el contexto europeo sólo los 
tratados de Artur Biedl (Wien, 1910) y Wilhelm Falta (Wien, 1913) precedieron al de Marañón (1914). Después llegarían los de Nicola Pende (Milán, 1916) y Julius Bauer (Berlín, 1927) ${ }^{7}$. Su primera revista especializada se publicaba tan sólo un año después de la francesa (Revue française d'endocrinologie, París, 1923); la de Biedl y Asher, Endokrinologie (Leipzig), llegaría en 1928, las más prestigiosas y difundidas. EI mundo norteamericano fue una excepción precoz con la figura de Charles de Sajous (1906-1912) que publicó su manual en 1902, fundó y presidió la Sociedad norteamericana en 1916 y en 1917 iniciaba Endocrinology de Los Ángeles. El mismo año se fundó la revista argentina y en 1922 la italiana, estas dos últimas de menor difusión. El mundo británico no contó con una revista especializada hasta 1939.

Aunque en Norteamérica la cátedra de "endocrinología aplicada" de la Universidad de Pennsylvania que ocupó Sajous data de 1921, las cátedras de endocrinología no abundaron en Europa, como revisaremos enseguida. Que sepamos, en la Facultad de Medicina de Bucarest se creó en 1933 la cátedra de endocrinología para Constantin Parhon ${ }^{8}$.

Pero más importante es que la obra de Marañón tuviera desde el principio una amplia difusión internacional. No sólo lo atestiguan las traducciones y reediciones de sus libros, algunos puestos al día en sus ediciones extranjeras. En 1929 se editaba en Budapest su original Praediabetische Zustände y publicó artículos originales en numerosas revistas médicas extranjeras, pero en las especializadas más prestigiosas lo hizo desde el momento de su aparición: en 1923 comenzó a publicar en la francesa que había nacido el año anterior y en 1929 en Endokrinologie, fundada en 1928. De 1921 data su primer artículo en la norteamericana Endocrinology.

Las publicaciones de Marañón -en castellano en su mayoría - alcanzaron una proyección internacional también circulando en los repertorios de bibliografía médica "en curso" de mayor difusión y en los especializados en endocrinología. Sólo hasta 1936 circularon 194 publicaciones de Marañón a través principalmente de Index Medicus (continuación del Index Catalogue of the Surgeon's General Office) y Quarterly Cumulative Index Medicus, precursores de Medline y su sucesor actual Pubmed. Contaba Marañón entonces con cerca de 660 publicaciones médicas originales (1909-1935) ${ }^{9}$; casi un tercio, pues, de su producción alcanzó difusión inmediata en la comunidad médica internacional de esta manera.

En su mayoría se trataba de publicaciones de endocrinología, metabolismo y nutrición, hasta 154, publicadas entre 1910 y $1935^{10}$, la mayoría artículos firmados en solitario y publicados en castellano ${ }^{11}$.
Aunque más de un tercio de esta producción endocrinológica difundida - hasta 60 trabajos - lo firmó Marañón en colaboración con 35 autores diferentes entre 1916 y $1935^{12}$. Y cincuenta y ocho trabajos, aparte de los libros citados, se habían publicado en el extranjero, la mayoría en revistas médicas generales -sobre todo las francesas y alemanas más prestigiosas - pero veinte en revistas endocrinología: nueve en la Revue française d'endocrinologie (1924-1935, tres en colaboración), cinco en Endokrinologie (19291934, tres en colaboración) y seis en Endocrinology, la única revista en inglés en la que publicó (1929-1934, en colaboración) ${ }^{13}$.

Pero la circulación internacional de publicaciones españolas de endocrinología hasta 1936 incluye las de cientos de autores de tres generaciones diferentes, entre ellos grandes productores como Enrique Fernández Sanz, José Gómez Ocaña, Enrique Carrasco Cadenas, Augusto Pi Sunyer, Carlos Jiménez Díaz, Juan Antonio Collazo, Eduardo Bonilla, José Morros Sardá, Carlos Blanco Soler o Torre Blanco, y la mayoría ocasionales entre los que se cuentan algunos tan prestigiosos como Amalio Gimeno, Ramón Turró, José Goyanes Capdevila, León Cardenal, Gustavo Pittaluga, Gonzalo Rodríguez Lafora, José Sanchis Banús, Nicolás Achúcarro, Juan Negrín, Carlos Sáinz de los Terreros, Vara López o Andrés Martínez Vargas, el grueso de cuya producción realizaban en su mayoría en otros campos $^{14}$. También figuran autores extranjeros que publicaron ocasionalmente en España, como Julius Bauer -en solitario y con Monguió. Sólo entre 1925 y 1932 circularon doce libros y 469 artículos españoles de endocrinología de 294 autores diferentes. Se publicaron en 36 revistas españolas y en 45 extranjeras -32 europeas y 13 americanas (Aguirre, 1986; 1991a y 1991b).

En las primeras bibliografías especializadas en endocrinología, las de Artur Biedl (1913; 1922), que fueron también la referencia internacional, la producción de Marañón ocupaba ya el tercer lugar en la edición de 1913, tras la del propio Biedl (16 años mayor que Marañón, había comenzado a publicar sobre endocrinología en 1895) y Falta (doce años mayor que Marañón, su primera publicación endocrina data de 1905), y lo mantuvo en la edición de 1922. En este momento se recogían ya 41 publicaciones suyas de endocrinología. De Biedl figuran 47, de Falta 41, de Bauer 27, también 27 de Parhon; 25 de Houssay, 21 de Pende, y 18 publicaciones de Sajous ${ }^{15}$. (Tablas 1 y 2).

Otros nueve autores españoles figuran en la edición de 1913 - José Gómez Ocaña, José Rodríguez Carracido, Juan Negrín López, Augusto Pi Sunyer, Ramón Turró Darder y los también catalanes Serrallach, Raventós Roigier, J. Alomar y Pedro Nubiola Espinós-, a los que se sumaron en la edición de 1922 al menos 
Tabla 1. Las publicaciones de las figuras iniciales de la endocrinología en la bibliografía especializada de Artur Biedl (1913)

\begin{tabular}{|l|c|c|c|}
\hline \multicolumn{1}{|c|}{ Autor } & $\begin{array}{c}\text { años de } \\
\text { publicación }\end{array}$ & en solitario & en colaboración* \\
\hline Artur Biedl (1869-1933) & $1895-1912$ & 21 & 12 \\
\hline Wilhelm Falta (1875-1950) & $1905-1913$ & 14 & 16 \\
\hline Gregorio Marañón (1887-1960) & $1909-1912$ & 17 & - \\
\hline Constantin I Parhon (1874-1969) & $1905-1911$ & 2 & 13 \\
\hline Nicola Pende (1880-1970) & $1903-1912$ & 14 & - \\
\hline Julius Bauer (1887-1979) & $1911-1913$ & 5 & 14 \\
\hline Charles E. Sajous (1852-1929) & $1906-1912$ & 5 & 6 \\
\hline Bernardo Houssay (1887-1971) & $1910-1912$ & 2 & 5 \\
\hline
\end{tabular}

*como primer firmante

Tabla 2. Las publicaciones de las figuras iniciales de la endocrinología en la bibliografía especializada de Artur Biedl (1922)

\begin{tabular}{|l|c|c|c|c|}
\hline \multicolumn{1}{|c|}{ Autor } & $\begin{array}{c}\text { años de } \\
\text { publicación }\end{array}$ & en solitario & en colaboración* & total \\
\hline Artur Biedl & $1895-1912$ & 33 & 14 & 47 \\
\hline Wilhelm Falta & $1905-1919$ & 24 & 17 & 41 \\
\hline Gregorio Marañón & $1909-1921$ & 36 & 4 & 40 \\
\hline Constantin I Parhon & $1905-1921$ & 3 & 24 & 27 \\
\hline Julius Bauer & $1911-1920$ & 20 & 7 & 27 \\
\hline Bernardo Houssay & $1910-1921$ & 15 & 10 & 25 \\
\hline Nicola Pende & $1903-1920$ & 19 & 2 & 21 \\
\hline Charles E. Sajous & $1906-1920$ & 17 & - & 17 \\
\hline
\end{tabular}

*como primer firmante

otros 37, además de los que firmaron sus trabajos con Marañón (Collazo, Rosique, Bonilla (también en solitario) y Pintos) ${ }^{16}$.

La obra británica de referencia en 1936 era el manual de endocrinología de H. D. Rolleston (Oxford, 1862-Londres, 1944), una titánica puesta al día que revisa la bibliografía retrospectiva hasta la más reciente -entre sus fuentes expresas la de Biedl (1913) y Endocrinology desde 1918. Cita en 19 ocasiones a Marañón a partir de doce publicaciones endocrinológicas suyas (1922-1935), sólo una de carácter histórico. El propio Rolleston relata su visita en 1935 a "la clínica del profesor Gregorio Marañón en Madrid", de donde toma un caso clínico para su obra y agradece a Marañón una de las ilustraciones que reproduce (Rolleston, 1936, 109; 363). La historia de la endocrinología del discípulo y asistente de Julius Bauer, Cornelius Medvei
(Budapest, 1905-Londres, 2000) puede considerarse la continuación de la obra de Rolleston donde la historia de los antecedentes se hipertrofia todavía más. Recoge sólo tres publicaciones de Marañón, pero le cita en ocho ocasiones, le dedica un epígrafe y una biografía independiente (Medvei, 1993, 217-218 y 453) basados casi exclusivamente en el trabajo de T.F. Glick (1976). También Medvei conoció a Marañón en Madrid a comienzos de la década de 1950, cuando tuvo ocasión de interesarse por José Monguió que se había formado con Bauer y él mismo en Viena (Medvei, 1993, 218).

La relación personal y profesional de Marañón con las grandes figuras iniciales mencionadas hasta ahora puede ejemplificarse en la incorporación a la edición de su revista de 1924 del que habría de ser premio Nobel en 1947, el argentino Bernardo Houssay, pero 
también en su participación en el libro homenaje publicado en Buenos Aires en 1935, o en el volumen conmemorativo dedicado a Wilhelm Falta (Marañón; Collazo, 1935) y en el dedicado a Parhon (Marañón; Almela; Collazo, 1934). A su vez, en 1929 presentaba la traducción al castellano del libro de endocrinología de Bauer con un prólogo que tituló "Endocrinología y constitución" y el año siguiente la traducción del Tratado de Falta con el prólogo "Veinte años de endocrinología". Nicola Pende prologaba en 1934 la edición italiana revisada por el propio Marañón de $L a$ evolución de la sexualidad y los estados intersexuales.

A excepción de Biedl y Houssay que se inclinaron a la patología experimental, las figuras internacionales citadas hasta ahora encabezaron la corriente denominada patología constitucional desde la medicina clínica, donde la endocrinología representó la posibilidad de abordar científicamente la dimensión personal de la enfermedad ${ }^{17}$. Julius Bauer, coetáneo de Marañón y considerado fundador de la patología constitucional con su tratado inaugural de 1917, fue el encargado de organizar el primer congreso internacional de endocrinología que debería haberse celebrado bajo su presidencia en Marienbad el verano de 1934. Cancelado a un mes de su inauguración por el gobierno nacional-socialista, se habría reunido sin duda en torno a los grandes clínicos como Pende, Falta, Marañón y el propio Bauer, a quienes la endocrinología había abierto las puertas no de la especialización, sino de la explicación endocrina de la salud y las enfermedades, del diagnóstico personal de cada uno de sus pacientes $y$, en consecuencia, la confianza en la posibilidad de prevenir las enfermedades individuales antes de que surgieran en cada persona. La radicalidad de la patología constitucional de Bauer se manifiesta en su propuesta de sustituir las enfermedades - especies morbosas- y "casos clínicos" por "el ser humano enfermo"; o en su rechazo y manifiesto desdén hacia la entonces "nueva" medicina psicosomática que regresaba a Europa desde la escuela de Chicago: no hay tal medicina psicosomática porque toda la patología es psicosomática. Su propuesta de que la Sociedad profesional se denominara "de endocrinología y genética" se debía a que ambas eran para Bauer los pilares de la constitución individual sobre la que podía asentar la enfermedad.

Nada lejos de estas posiciones, en 1934 Marañón escribía que la enfermedad "se acoplaba a la persona", y también que "de las estadísticas clínicas pasamos a los casos individuales de nuevo, de los síntomas y signos a la explicación patogénica que integra todos y cada uno de los factores individuales y personales pasados y presentes, del diagnóstico de la enfermedad bien establecida a su prevención" (Marañón, 1935a, 30).
$Y$ sin embargo, cuando finalmente se organizaba en 1957 el primer congreso internacional que habría de celebrarse en 1960 en Copenhague, esta manera de entender la endocrinología no estuvo presente allí. Ni Bauer, ni Pende, ni Marañón ni sus escuelas participaron. Bernardo Houssay presidió el congreso y el español fue en consecuencia el idioma oficial junto con el inglés de la organización británica. Las cosas habían cambiado mucho bajo la influencia de los grandes logros de la fisiología y patología experimentales.

Esa ya no era la endocrinología de Marañón, aunque venía explicando las enfermedades de las glándulas de secreción interna desde 1931 en su cátedra de doctorado. Cuando se creó la cátedra de endocrinología precisamente para Marañón, además de la polémica - miserable, por política - en el seno de la Academia Nacional de Medicina (Granjel, 2008) para emitir el informe requerido, normalmente un trámite sin más, resultó polémica también la emisión del informe de la Facultad de medicina, otro mero trámite, aunque por razones muy diferentes. Los profesores más cercanos a Marañón creían que debía ocupar la cátedra vacante de Patología médica. Entendían con el propio Marañón que la endocrinología no era una parte escindible de la patología sino la doctrina integradora y central para explicar cualquier enfermedad y que, en consecuencia, Marañón debía explicar patología y clínica médicas, en lo que era un verdadero maestro. También podemos entender que además de satisfacer mejor las convicciones científicas de Marañón, en esos momentos hubiera preferido no ser nombrado directamente, aunque fuera lo legalmente establecido para una cátedra de doctorado. Como temía, sus detractores por esas fechas vieron en la cátedra un premio a su activa participación en la gestación y triunfo de la República.

Como mencionamos, las cátedras de endocrinología escasearon en Europa. Las grandes figuras como Falta, Pende o Bauer enseñaron patología y clínica médica. Al igual que Marañón fueron internistas con una clara orientación clínica quienes impulsaron en Europa la endocrinología. Falta, Marañón y Pende jamás modificaron el rótulo de sus Institutos de $\mathrm{Pa}$ tología médica; Julius Bauer siguió enseñando clínica médica tras su exilio a Estados Unidos en 1938 y hasta su muerte.

Conociendo estas posiciones científicas de Marañón podemos entender que en 1935, resumiendo los veinticinco años de labor en el Hospital provincial, escribiera que cuando su generación "empezó a trabajar en sentido moderno estábamos en la situación de Robinson Crusoe que tuvo que ser albañil, cazador, cocinero, maestro y público de sí mismo" (Marañón, $1935 a, 6)^{18}$ y que sólo reconociera como maestros directos a sus profesores Manuel Alonso Sañudo, cate- 
drático de patología médica, Alejandro San Martín, de cirugía, y a Juan Madinaveitia, agregado de Sañudo ${ }^{19}$, además de, por supuesto, a Cajal (Marañón, 1935a, $5-6 ; 17-48)$. Se refería sin duda a la nueva medicina atenida rigurosamente al método positivo tanto en la clínica como en el laboratorio que enseñaban Sañudo y Madinaveitia y, entre otras cosas, el respeto a las personas y en particular a los pacientes de Alejandro San Martín. De haberse referido a la endocrinología habría reconocido sin duda la labor previa de su profesor de fisiología José Gómez Ocaña (1860-1919) que explicaba secreciones internas en 1895 y publicó, además de sus lecciones (1895a), una de las primeras exposiciones de conjunto sobre las secreciones internas, aunque como introducción a su difundido libro sobre la glándula tiroides (1985b); o a Enrique Fernández Sanz (1872-1950) que fue posiblemente el primero en explicar endocrinología $-\mathrm{y}$ publicar después sus lecciones (1912) - en la cátedra de Patología médica de Alonso Sañudo. Y, por qué no, a la escuela de Ramón Turró (1854-1926) que desarrollaba su labor pionera en Cataluña, desde la fisiología experimental. La endocrinología fue para Marañón un medio, revolucionario pero medio al fin y al cabo, para conocer la enfermedad y diagnosticar y tratar adecuadamente a sus enfermos.

Aunque no se vieran cumplidas las expectativas que depositaron Marañón y su generación en la endocrinología como una revolución médica que permitiría explicar científicamente, al fin, la dimensión personal de la enfermedad, la corriente que conformaron sí contribuyó decisivamente a la incorporación del sujeto a la patología actual. Y a la vez, fueron los responsables, aun sin quererlo, de la constitución de la endocrinología en una especialidad médica.

\section{NOTAS}

1 Desde Granjel (1960), han abordado también contenidos de su obra endocrinológica Botella Llusiá (1964; 1972; 2003, 429-446); Botella Llusiá; Fernández de Molina, eds., (1997); Fernández de Molina (2003, 349-369). Dedicados exclusivamente a la obra endocrinológica de Marañón, López Vega (2009 b) y Clavero (2010).

2 Especialmente fijada en el mundo de habla inglesa desde Medvei (1982, 774-75; 1991, 217-18 y 453), tras Glick (1976), sitúa a Marañón en el panteón de los padres de la endocrinología por su singular esfuerzo y valía personales, a pesar de que el propio Glick (1984) ha abordado el peso y la proyección internacional de la escuela fisiológica catalana. Apunta la contribución clínica catalana y la de otros grupos españoles, como el gallego, Orozco (1999). Ponte (2009) está abordando la contribución de Nóvoa Santos y su grupo de Santiago de Compostela. López Vega (2009b) sitúa la labor endocrinológica de Marañón en la renovación científica española del primer tercio del siglo XX con gran acierto.

3 Sobre la "Edad de Plata" (1898-1936) de la cultura española existe una abundante bibliografía, lo mismo que sobre la generación europea de 1914. Albarracín (1993) ofrece una síntesis de la medicina española de esas tres generaciones. En lo que concierne a la medicina de Marañón sigue resultando indispensable la obra de Laín (1969) $y$, recientemente, Balaguer; Ballester (2010).
4 Aguirre Marco (1990) y (1993) a propósito de la patología tiroidea.

5 La polémica en la prensa diaria, descrita por Glick (1976). Sobre la tendencia a la panacea de la opoterapia tiroidea en España en las últimas décadas del siglo XIX -de base empírica o racional pero no científica todavía (Ackerknecht, 1962)Aguirre (2000).

6 Lellep (1990) le dedica el libro por su enorme labor sanitaria en varias regiones bociógenas españolas que terminó abruptamente con la guerra civil, al final de la cual se pierde su rastro.

7 Artur Biedl (Viena, 1869-1933): Wilhelm Falta (Karlsbad, 1875-Viena, 1950); Julius Bauer (Nachod, 1887- Los Ángeles (CA), 1979); Nicola Pende (Noicattaro, Bari, 1880-Roma, 1970).

8 Constantin I. Parhon (Câmpulung Muscel, Arges, 1874-Bucarest, 1969), que habría de presidir el primer gobierno comunista rumano tras la segunda guerra mundial, también fundó la sociedad rumana y su revista Acta endocrinologica en 1938. Pero él mismo formaba parte del consejo de redacción de Revue française d'endocrinologie y su propia obra y la de sus colaboradores se difundió sobre todo en francés. Su manual data de 1909 (Bucarest), aunque lo que circuló fue un resumen en francés publicado ese mismo año en París.
9 Para la producción original de Marañón utilizamos la reciente bibliografía de Lopez Vega (2009a) que revisa todas las anteriores. No hemos contabilizado los prólogos si no se publicaron aparte, ni las críticas bibliográficas. Para la producción original de Marañón y la de su escuela en este periodo sigue resultando imprescindible la bibliografía de Veinticinco años de labor (Marañón, 1935a, 51-326).

10 Para la elaboración de estas cifras hemos utilizado los repertorios de una serie de tesis de licenciatura y doctorales sobre la aportación española a las publicaciones que circularon en la comunidad médica internacional dirigidas por José María López Piñero en la Universidad de Valencia: Aguirre, 1986; Aleixandre, 1991; Carbonell, 1986; Herrero, 1990; Salavert Nadal, 1983 y Sanchis, 1987 (su contexto en López Piñero; Terrada Ferrandis (1993). Por razones obvias, las publicaciones que citemos a partir de ahora sin atender a sus contenidos no constarán en la bibliografía. Sus referencias completas están accesibles en estos repertorios y en los de la nota previa.

11 Cinco libros originales publicados en Madrid excepto uno en Budapest (1927); otros cinco son traducciones de La Edad Crítica al alemán (Heidelberg, 1928) y al inglés (St. Louis, 1929) y de La evolución de la sexualidad y los estados intersexuales en la especie humana al inglés, al francés y al italiano (Londres, 1932; París, 1934; Bolonia, 1934 respectivamente). 
12 La primera colaboración recogida es de 1911 (con García Urdiales) pero la primera de endocrinología data de 1916, publicada en París con J.G. Pinto. Los autores con los que colaboró en más de dos publicaciones fueron J.A. Collazo con quien firmó 23; cinco firmó con José Morros Sardá; cuatro con Eduardo Bonilla, tres con Álvarez Cascos y otros tres con Ossorio Florit. Ocho autores diferentes firmaron con él dos trabajos y 21 autores colaboraron en una sola ocasión. Sólo seis trabajos firmó en segundo o tercer lugar, tras Rodríguez Lafora (1915), Garma (1927), Collazo (1933-1934) y de la Villa (1934). También como segundo firmante circularon el tratado de medicina interna que dirigió con Teófilo Hernando (1916) y una publicación con José Sanchis Banús y Pío del Río-Hortega sobre la enseñanza de la medicina, que obviamente no contabilizamos entre los de endocrinología.

13 Del total de trabajos publicados en el extranjero, circularon treinta en francés, once en alemán, nueve en inglés, siete en castellano, y uno en italiano. Las revistas médicas generales incluyen ocho francesas, una belga, cinco alemanas, una de Viena, seis latinoamericanas y una italiana, entre ellas las más prestigiosas de medicina interna.

14 Un panorama general sobre su labor $y$ sus relaciones profesionales e institucionales en el conjunto de la medicina española de la época en Albarracín (1993).

15 Dado que se trata de bibliografías retrospectivas elaboradas con criterios amplios, en ellas figuran productores mayores que los aquí citados, bien por ser muy anteriores, bien por la procedencia de sus publicaciones especialmente evidente en el mundo francés de "Comptes rendus"-, bien por su dedicación a otros campos como la morfología, la zoología, la criminología, o la fisiología. Ofrecemos las cifras de las figuras iniciales de la especialidad médica propiamente dicha según los criterios expuestos de partida.

16 Figuran entre los más conocidos Teófilo Hernando, Rodríguez Lafora, Sanchis Banús, Gustavo Pittaluga y Leandro Cervera, que también firmó con Houssay como Carulla Riera. De la escuela catalana también, las publicaciones de P. Domingo y S. Vilaseca, entre otros. Goyanes Capdevila, Alday Redonet, Blanc Fortacín, Sáinz de los Terreros, Agustín del Cañizo -uno de los primeros becados de la JAE-, Luis Guilera Molas ... no podemos mencionarlos a todos aquí. Cabe decir que a partir de 1919 Biedl utilizó también la revista Endocrinology para elaborar su repertorio y que numerosas publicaciones españolas proceden de esa fuente. La mayoría de las obras difundidas se publicaron en España.

17 Estudiado especialmente por Laín Entralgo (1950, 489-724, y 1976).

18 Sin embargo no desaprovechó ocasión alguna para reconocer la labor de la generación precedente que es la que les posibilitó hacer una ciencia básica y una medicina a la altura de la mejor europea, así como establecer las relaciones internacionales adecuadas. Ese mismo año escribía de Amalio
Gimeno (1850-1936) que "acaso los médicos actuales, ya en posesión de centros de investigación adecuados, olvidan a aquéllos que los crearon y pusieron en marcha, en beneficio de las generaciones futuras, todo lo que, sin duda, no ha surgido por generación espontánea, sino por el esfuerzo de otros hombres a los que debemos gratitud» (1935b, 12). Recordemos por ejemplo que de la creación de la Junta para la Ampliación de Estudios fueron responsables directos San Martín y Gimeno al frente sucesivamente del Ministerio de Instrucción pública de diferentes gobiernos de Alfonso XIII. También lo ocupó Carlos María Cortezo (1850-1933) que con Amalio Gimeno y Ángel Pulido (1852-1932) son considerados las tres grandes figuras de la salud pública española moderna. Pulido había afirmado en 1908 que la mejor medicina para las Hurdes era sin duda la carretera (Marañón, 1927b, 64). Pero, como es sabido, Marañón consiguió comprometer al Estado en la solución de ese mismo problema, como consiguió también la edificación de un hospital adecuado para los enfermos infecciosos del provincial cuando hasta entonces las mejoras de las salas o los laboratorios habían salido del bolsillo de Juan Madinaveitia, de Luis Simarro y del propio Marañón en sus inicios. No cabe duda de que la conciencia por los problemas sociales se agudizaba también en España en la década de 1930, lo mismo que arraigaba el concepto de "Estado benefactor" que señalara Rosen (1974).

19 Alejandro San Martín y Satrústegui (1847-1908); Manuel Alonso Sañudo, (1856-1912) y Juan Madinaveitia y Ortiz de Zárate (1861-1938).

\section{BIBLIOGRAFÍA}

Ackerknecht, E.H. (1962). Aspects of the history of therapeutics. Bull. Hist. Med., 36, 389-419.

Aguirre Marco, C. P. (1986). La aportación española a las publicaciones sobre endocrinología circulantes en la comunidad médica internacional, 1925 1932. Inventario y estudio bibliométrico. Valencia, Tesis de licenciatura.

Aguirre Marco, C. P. (1991a). Sobre la aportación española a las publicaciones de endocrinología que circularon en la comunidad médica internacional, 1925-1932. Actas del V Congreso de la Sociedad española de Historia de las
Ciencias y las técnicas, Murcia, 1991, vol. II, p. 1251-1264.

Aguirre Marco, C. P. (1991b). Las publicaciones españolas sobre la glándula tiroides hasta 1940. Fuentes y materiales. Actas del IX Congreso Nacional de Historia de la medicina, Zaragoza, 1989, vol. III, p. 827-832.

Aguirre Marco, C. P. (1993). La glándula tiroides en la medicina española del siglo XIX. Valencia, tesis doctoral. (Mff)

Aguirre Marco, C.P. (1994). Los conocimientos sobre la glándula tiroides

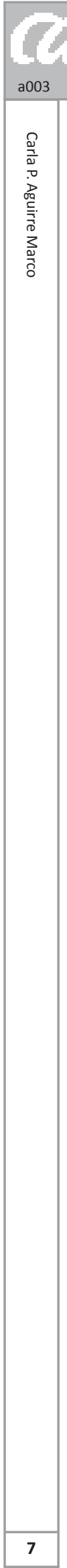

en los tratados de patología y clínica internas utilizados en la formación de los médicos españoles durante el siglo XIX. En H. Capel Sáez, J. M. López Piñero, J. Pardo Tomás (Coords.) (1991; 1994), Ciencia e ideología en la ciudad. Valencia, Conselleria de Obras Públicas y Urbanismo, 2 vols. Vol 1, pp. 175-209.

Aguirre Marco, C.P. (2000). Del medicamento específico a la panacea. la introducción de la tiroidoterapia en España, 18931898. Actes de les $\vee$ Trobades d'història de la Ciència i de la tècnica, Observatori de l'Ebre, 11-13 desembre 1998, Barcelona, SCHCT, pp. 253-258. 
Albarracín Teulón, A. (1993). Las ciencias médicas. En P. Laín Entralgo (Coord.), La Edad de Plata de la cultura española (1898-1936), Madrid: Espasa Calpe, 2 vols. Historia de España, vol. 39 (2), 495-544.

Aleixandre, R. (1991). La aportación española a las publicaciones circulantes en la comunidad médica internacional 1933-1935. Valencia, tesis doctoral.

Balaguer, E. y Ballester, R. (2010). Marañón y la medicina en España. En Marañón, 1887-1960. Médico, humanista y liberal, Madrid, Sociedad Estatal de Conmemoraciones Culturales, ed., 8195.

Bauer, J. (1917). Die konstitutionelle Disposition zu inneren Krankheiten. Berlin: Springer (eds. castellanas (1930): Herencia y constitución, Jimena F. de la Vega, trad., Barcelona, Manuel Marín y (1944): Constitución y enfermedad: patología constitucional aplicada, Emilio Mira López, trad., Barcelona, López).

Bauer, J. (1927). Innere Sekretion. Berlin: Springer. (Trad. castellana, (1929): Fisiología, patología y clínica de las secreciones internas, Madrid, tip. Artística).

Biedl, A. (1910). Innere Sekretion: ihre physiologischen Grundlagen und ihre Bedeutung für die Pathologie. Wien, Urban \& Schwarzenberg, 1910. (2- ed. 1913; 4a ed., 1922, 4 vols.).

Biedl, A. (1913). Literatur. En Innere Sekretion: ihre physiologischen Grundlagen und ihre Bedeutung für die Pathologie. Zweite Aufl. Wien, Urban \& Schwarzenberg, 392-653.

Biedl, A. (1922). Literatur. En Innere Sekretion: ihre physiologischen Grundlagen und ihre Bedeutung für die Pathologie. Wien, Urban \& Schwarzenberg. Vierte Aufl. Band 3, 1-480.

Botella Llusiá, J. (1972). Gregorio Marañón. El hombre, la vida, la obra. Publicaciones del Centro Universitario de Toledo.

Botella Llusiá, J. (1964). Comentarios a la obra endocrinológica de Marañón. En Hospital general, marzo-abril, 1964.

Botella Llusiá, J., Fernández de Molina, A. (Eds.) (1997). La Evolución de la Sexualidad y los Estados Intersexuales. Madrid: FGM-Díaz de Santos.
Botella Llusiá, J. (2003). Marañón: Una nueva visión de la medicina. En ROOM, 20-24.

Carbonell Fornet, L. (1986). La aportación española a las publicaciones circulantes en la comunidad médica internacional durante el decenio 1903-1912. Inventario, bibliometría y thesaurus. Valencia, Tesis doctoral.

Clavero, J. A. (2010). La obra endocrina de Marañón. En Marañón, 1887-1960. Médico, humanista y liberal. Madrid: Sociedad Estatal de Conmemoraciones Culturales, 99-111.

Falta, W. (1913). Die Erkrankungen der Blütdrüsen. Wien: Springer. (Trad. Castellana: Tratado de las enfermedades de las glándulas de secreción interna Barcelona, Labor, 1930)

Fernández de Molina, A. (2003). Contribución de Marañón a la teoría de la emoción. En ROOM, 349-371.

Fernández Sanz, E. (1912). Enfermedades de las glándulas de secreción interna. Lecciones explicadas en la Facultad de Medicina de Madrid. Madrid, F. Beltrán.

Glick, T.F. (1976). On the difusión of a new specialty: Marañon an the crisis of Endocrinology in Spain. Journal of the history of biology, 9, 287-300.

Glick, T.F. (1984). Walter B. Cannon la ciència catalana d'entreguerres. Ciència, en-feb, 40-51.

Gómez Ocaña, J. (1895a). Secreciones internas. Lecciones de fisiología explicadas en la Facultad de Medicina de Madrid. El siglo medico, 42, 84-86; 99-101.

Gómez Ocaña, J. (1895b). Nuevas investigaciones sobre el tiroides y la medicación tiroidea. Madrid: N. Moya/ Asilo de huérfanos.

Granjel, L.S. (1960). Gregorio Marañón, su vida y su obra. Madrid: Guadarrama.

Granjel, L.S. (2008). La Academia “años treinta" (Política y medicina). Anales de la Real Academia Nacional de Medicina, 1, 79-90.

Herrero Cucó, L.A. (1990). La aportación española a la literatura circulante en la comunidad médica internacional durante el periodo 1913-1925. Valencia, tesis doctoral.
Laín Entralgo, P. (1950). La historia clínica. Historia y teoría del relato patográfico. Madrid: CSIC.

Laín Entralgo, P. (1969). Gregorio Marañón. Vida, obra y persona. Madrid: Espasa Calpe.

Laín Entralgo, P. (1979). Subjetividad, subjetualidad y enfermedad. Realitas, 3-4 (1976-1979) Trabajos del seminario Xavier Zubiri, Madrid, 45-78. (También en (1986): Ciencia, técnica y medicina, Madrid, Alianza, 284-314).

Lellep, R. (1990). A simple matter of salt. University of California Press.

López Piñero, J.M; Terrada, M.L. (1993). Veinte años de investigación bibliométrica. Valencia: Instituto de Estudios Documentales e Históricos sobre la Ciencia (CSIC-Universidad de Valencia).

López Vega, A. (2009a). Gregorio Marañón y el nacimiento de la endocrinología en España: ejemplo ilustrativo del impulso científico del primer tercio del siglo XX español. Circunstancia. Revista de Ciencias Sociales del Instituto Universitario José Ortega y Gasset, Año VII, no 19, Mayo 2009.

López Vega, A. (2009b). Biobibliografía de Gregorio Marañón. Madrid: Dykinson (Biblioteca del Instituto Antonio de Nebrija de estudios sobre la universidad, n. 19) http://hdl.handle. net/10016/6178

Marañón, G. (1914). Las glándulas de secreción interna y las enfermedades de la nutrición. Madrid, Ruiz (2ª ed., 1916; 3a ed., 1920).

Marañón, G. (1915). La doctrina de las secreciones internas. Su significación biológica y sus aplicaciones a la clínica. Madrid: Corona.

Marañón, G. (1920). Las enfermedades de las glándulas de secreción interna (Endocrinología). En Hernando, T.; Marañón, G., dirs. (1916-1934), Manual de medicina interna. Madrid: Ruiz, 3 vols. Vol. 2, 3-108.

Marañón, G. (1922). Problemas actuales de la doctrina de las secreciones internas. Madrid: Ruiz.

Marañón, G. (1927a). Praediabetische Zustände. Budapest: Novak.

Marañón, G. (1927b). El bocio y el cretinismo. Madrid: Páez. 
Marañón, G. (1929). Endocrinología y constitución. En J. Bauer: Fisiología, patología y clínica de las secreciones internas. Madrid: tip. Artística, I-XVI.

Marañón, G. (1930). Veinte años de endocrinología. En W. Falta: Tratado de las enfermedades de las glándulas de secreción interna, I. Bofill y L. Girones trads., de la segunda ed. alemana, Barcelona, Labor, i-xv. (También (1931): "Veinte años de endocrinología. Lección inaugural del curso de Endocrinología en la Facultad de Medicina de Madrid", en Medicina Ibera, 25, 347).

Marañón, G. (1934). L'evoluzione della sessualità é gli stati intersessuali, Edz. riveduta dell autore. Con prefazione del prof. Nicola Pende. Bologna: Zanichelli.

Marañón, G. (1935a). Veinticinco años de labor. Historia y bibliografía de la obra del prof. Gregorio Marañón y del Instituto de Patología Médica de Hospital de Madrid. Recogida por sus discípulos. Madrid: Espasa Calpe.

Marañón, G. (1935b). Don Amalio Gimeno, médico. En Real Academia Nacional de Medicina; F.J. Cortezo, ed., Antología de trabajos científicos, literarios, políticos y sociales del profesor Amalio Gimeno y Cabañas, primer Conde de Gimeno, publicada con ocasión del 85 aniversario de su nacimiento. Madrid, imp. J. Cosano, 1-12.

Marañón, G. (1935c). "La vida sexual en la insuficiencia suprarrenal", en Libro homenaje al profesor Houssay, Buenos Aires.

Marañón, G.; Almela, J. y Collazo, J.A (1934). Le chimisme musculaire dans le diabete humain et expérimental. En Vol.
Jubilaire en honneur du Pro. C. Pahon, lassy (Romania).

Marañón, G. y Collazo, J. A. (1935). “Die Deshydrierung in der Addisonschen Krankheit und ihr Mechianismus. En Sonderabdruck aus Wiener Archiv für Innere Medizin, Urban und Schwarzenberg, Wien, 189-200.

Marañón, G. (1939). Manual de enfermedades endocrinas $y$ del metabolismo. Buenos Aires: Hechette.

Medvei, V.C. (1982). A History of Endocrinology. London: MTP. (reimp., 1984).

Medvei, V.C. (1993). A History of Clinical Endocrinology. London-New York: Parthenon.

Orozco Acuaviva, A. (1999). Historia de la endocrinología española. Madrid: Díaz de Santos.

Parhon, C.I. y Goldstein, M. (1909). Secrețiile Interne. Bucarest. (Resumen (1909): Secretion interne, París).

Pende, N. (1916). Endocrinologia: patologia e clinica degli organi a secrezione interna. Milano, Vallardi. (2a ed., 1920; 4a ed., 19342 vols.; 5a ed, 1949) (Trad. castellana (1939): Endocrinología: patología y clínica de los órganos de secreción interna, Barcelona, Salvat).

Ponte Hernando, F. J. (2009). Roberto Nóvoa Santos (1885-1933) precursor en el tratamiento de la diabetes con incretinas. Santiago de Compostela: Nilo imp.

Portela Arcos, A. (1986). La aportación española a la literatura circulante en la comunidad médica internacional durante los periodos 1900-19001 y 1925-26. Valencia, tesis de licenciatura.

ROMM. FGM (2003). Revisión de la obra médica de Marañón. Puertollano, Ciudad Real. http://www.fund-gregoriomaranon.com/

Rolleston, H.D. (1936). The endocrine organs in health and disease. With a historical review. London: Oxford University Press.

Rosen, G. (1944). The specialization of medicine. New York: Froben Press.

Rosen G. (1974). Análisis genético del concepto de medicina social. En G. Rosen (1985), De la policía médica a la medicina social. Mexico: siglo XXI, 125.

Sajous, C.E. de (1903; 1907). The Internal secretions and the principles of medicine. Philadelphia, FA Davies Co. 2 vols.

Salavert Nadal, M.T. (1983). La aportación española a la literatura circulante en la comunidad médica internacional durante el quinquenio 1921-25. Valencia, tesis de licenciatura.

Sanchis Fortea, M. (1987). La aportación española a las publicaciones sobre diabetes mellitus circulantes en la comunidad médica internacional, 18241975. Valencia, tesis doctoral.

Vaticón, D.; Botella Llusiá, J. et al. (2003). Marañón y la biología sexual: el nacimiento de la sexología en España. En ROOM, 161-213. 